\title{
Ctyokine's role in autoimmune melee probed
}

Immunologists have paid a lot of attention lately to the T helper type $17\left(T_{H} 17\right)$ cytokine IL-17, which is thought to be central to the pathogenesis of multiple sclerosis and other autoimmune diseases. But new findings by Haak et al. ${ }^{1}$ could take some of the sheen off of this molecule. Studying a mouse model of multiple sclerosis, experimental autoimmune encephalomyelitis (EAE), the researchers found that a major form of interleukin-17 (IL-17), IL-17A, did not exacerbate disease when overexpressed specifically in T cells. Moreover, genetic and antibody-blocking experiments suggest that mice lacking the function of both IL-17A and another isoform, IL-17F, show disease with similar incidence and severity as mice with these molecules. The findings emerge against a backdrop of other studies, such as two last year in the Journal of Experimental Medicine ${ }^{2,3}$, which put some emphasis onto other players in autoimmunity. Is IL-17 now heading out of fashion? We asked three experts their views.

\section{Lars Fugger:}

The study by Haak et al. ${ }^{1}$ adds to the emerging evidence that IL-17 is not the deus ex machina in autoimmune central nervous system disease after all. This is hardly surprising, as previous lessons in this field have repeatedly demonstrated that there are no magical solutions to the immunopathology of complex diseases like multiple sclerosis. It is, nevertheless, very important not to jump to the opposite conclusion, that IL-17 has no role at all in multiple sclerosis, as the animal studies do not necessarily reproduce the human disease, and IL-17 is clearly present in brain lesions from people with multiple sclerosis. The ultimate test of whether IL-17 plays a part in multiple sclerosis will come from clinical trials in which participants are treated with drugs that block its action.

Lars Fugger, Professor of Neuroimmunology, Oxford University, Oxford, UK.

\section{Joan Goverman:}

Haak et al. ${ }^{1}$ provide convincing evidence that the two main isoforms of IL-17 are not required for actively induced EAE-in this combination of mouse strain and disease-inducing antigen. However, their conclusion that "neither of these $\mathrm{T}_{\mathrm{H}} 17$ cytokines are key players in EAE" should be treated cautiously.

Previous work suggests that both $\mathrm{T}_{\mathrm{H}} 1$ and $\mathrm{T}_{\mathrm{H}} 17 \mathrm{~T}$ cell subsets are pathogenic ${ }^{2,3}$. We have previously shown that the epitope used by Haak et al. ${ }^{1}$ to induce disease is a naturally $\mathrm{T}_{\mathrm{H}} 1$-biased epitope ${ }^{7}$, so it is not surprising that IL-17 contributes little to the pathogenicity of $\mathrm{T}$ cells primed by this epitope. Other epitopes are more $\mathrm{T}_{\mathrm{H}}$ 17-biased, and the relative abundance of each subset influences the site of inflammation more than incidence of disease ${ }^{7}$.

The collective findings from animal models suggest that the role of IL-17 and the primary cytokine secreted by $\mathrm{T}_{\mathrm{H}} 1$ cells, interferon- $\gamma$, may vary depending on the nature of the autoimmune response of the individual, sounding a note of caution for multiple sclerosis therapy development.

Joan Goverman, professor of Immunology, University of Washington, Seattle, Washington, USA.

\section{The ultimate test of whether IL-17 plays a} part in multiple sclerosis will come from clinical trials._- Lars Fugger

\section{Thomas Korn and Vijay Kuchroo:}

The findings of Haak et al. ${ }^{1}$ contrast with other research showing a role for IL-17 in autoimmunity. For instance, IL-17A has a direct effect on endothelial cells of the blood-brain barrier ${ }^{4}$, and IL-17-specific antibody can suppress immunopathology in other animal models of autoimmunity ${ }^{2,5}$. Moreover, treatment with a neutralizing antibody to IL-17A is showing remarkable efficacy in human autoimmune diseases (D. Patel, Novartis Institutes for Biomedical Research, personal communication).

Haak et al. ${ }^{1}$ deserve credit for highlighting that $\mathrm{T}_{\mathrm{H}} 17$ cells are not equivalent to IL-17. Aside from IL-17A and IL-17F, $\mathrm{T}_{\mathrm{H}} 17$ cells also produce IL-21, IL-22 and possibly other factors that contribute to autoimmunity. After their initial differentiation by transforming growth factor- $\beta$ plus IL-6, $\mathrm{T}_{\mathrm{H}} 17$ cells are expanded and matured by IL-23. Accumulating evidence demonstrates that IL-23 receptor variants control susceptibility to a number of human autoimmune diseases ${ }^{6}$, providing genetic evidence for the role of the IL-23- $\mathrm{T}_{\mathrm{H}} 17$ axis in human autoimmunity.

Despite the new findings, it remains likely that IL-23- $\mathrm{T}_{\mathrm{H}} 17-$ driven immune responses have a crucial role in inducing tissue damage in autoimmune diseases, although the relative pathogenic impact of the IL-23- $\mathrm{T}_{\mathrm{H}} 17$ pathway versus the IL-12- $\mathrm{T}_{\mathrm{H}} 1$ pathway might vary depending on how effector $\mathrm{T}$ cells are induced ${ }^{2,3}$ and on whether the target organ is susceptible to IL-17- or IFN$\gamma$-mediated tissue inflammation.

Thomas Korn, fellow in the Department of Neurology, Technical University Munich, Munich, Germany and Vijay Kuchroo, Professor of Neurology, Harvard Medical School, Boston, Massachusetts, USA. 